\title{
Undernutrition and severe acute malnutrition in children
}

\author{
(@) $(\mathcal{Q} \Theta$ OPEN ACCESS
}

This article's (BMJ 2017;359:j4877, doi:10.1136/bmj.j4877) infographic and appendix 1 have been amended to correct the description of the WHO appetite test. Appendix 2 had a wrong reference, and this has also been corrected. 